Published in final edited form as:

Acta Diabetol. 2016 June ; 53(3): 439-447. doi:10.1007/s00592-015-0815-z.

\title{
Analysis of the Relationships between Type 2 Diabetes Status, Glycemic Control, and Neuroimaging Measures in the Diabetes Heart Study Mind
}

\author{
Laura M. Raffield ${ }^{1,2,3}$, Amanda J. Cox ${ }^{4}$, Barry I. Freedman ${ }^{5}$, Christina E. Hugenschmidt ${ }^{6}$, \\ Fang-Chi Hsu ${ }^{7}$, Benjamin C. Wagner ${ }^{8}$, Jianzhao $\mathrm{Xu}^{2,3}$, Joseph A. Maldjian ${ }^{8}$, and Donald W. \\ Bowden $^{2,3,9,10}$ \\ ${ }^{1}$ Molecular Genetics and Genomics Program, Wake Forest School of Medicine, Winston-Salem, \\ NC, USA \\ ${ }^{2}$ Center for Human Genomics, Wake Forest School of Medicine, Winston-Salem, NC, USA \\ ${ }^{3}$ Center for Diabetes Research, Wake Forest School of Medicine, Winston-Salem, NC, USA \\ ${ }^{4}$ Molecular Basis of Disease, Griffith University, Southport, QLD, Australia \\ ${ }^{5}$ Department of Internal Medicine - Nephrology, Wake Forest School of Medicine, Winston-Salem, \\ NC, USA \\ ${ }^{6}$ Department of Gerontology and Geriatrics, Wake Forest School of Medicine, Winston-Salem, \\ NC, USA \\ ${ }^{7}$ Department of Biostatistical Sciences, Wake Forest School of Medicine, Winston-Salem, NC, \\ USA \\ ${ }^{8}$ Department of Radiology, Wake Forest School of Medicine, Winston-Salem, NC, USA \\ ${ }^{9}$ Department of Biochemistry, Wake Forest School of Medicine, Winston-Salem, NC, USA \\ ${ }^{10}$ Center for Genomics and Personalized Medicine Research, Wake Forest School of Medicine \\ Medical Center Boulevard, Winston Salem, NC, USA, 27157
}

\section{Abstract}

Aims-To examine the relationships between type 2 diabetes (T2D) status, glycemic control, and T2D duration with magnetic resonance imaging (MRI)-derived neuroimaging measures in European Americans from the Diabetes Heart Study (DHS) Mind cohort.

Methods-Relationships were examined using marginal models with generalized estimating equations in 784 participants from 514 DHS Mind families. Fasting plasma glucose, glycated hemoglobin, and diabetes duration were analyzed in 682 participants with T2D. Models were

Tel: +1 336-713-7507; ; Email: dbowden@wakehealth.edu

Conflicts of Interest All authors report no conflicts of interest.

Ethical approval: All procedures performed in studies involving human participants were in accordance with the ethical standards of the institutional and/or national research committee and with the 1964 Helsinki declaration and its later amendments or comparable ethical standards.

Informed consent: Informed consent was obtained from all individual participants included in the study. 
adjusted for potential confounders, including age, sex, history of cardiovascular disease, smoking, educational attainment, and use of statins or blood pressure medications. Association was tested with gray and white matter volume, white matter lesion volume, gray matter cerebral blood flow, and white and gray matter fractional anisotropy and mean diffusivity.

Results-Adjusting for multiple comparisons, T2D status was associated with reduced white matter volume $\left(\mathrm{p}=2.48 \times 10^{-6}\right)$ and reduced gray and white matter fractional anisotropy $(\mathrm{p} \unlhd 0.001)$ in fully adjusted models, with a trend towards increased white matter lesion volume $(\mathrm{p}=0.008)$ and increased gray and white matter mean diffusivity ( $\mathrm{p} \unlhd$ ).031). Among T2D affected participants, neither fasting glucose, glycated hemoglobin, nor diabetes duration were associated with the neuroimaging measures assessed ( $\mathrm{p}>0.05)$.

Conclusions-While T2D was significantly associated with MRI-derived neuroimaging measures, differences in glycemic control in T2D affected individuals in the DHS Mind study do not appear to significantly contribute to variation in these measures. This supports the idea that presence or absence of T2D, not fine gradations of glycemic control, may be more significantly associated with age-related changes in the brain.

\section{Keywords}

Type 2 diabetes; magnetic resonance imaging; glycemic control; Diabetes Heart Study

\section{Introduction}

Type 2 diabetes (T2D) has been associated both with mild, age-related decrements in cognitive testing performance and with increased risk of Alzheimer's disease and vascular dementia [1-3]. This is reflected in changes in magnetic resonance imaging (MRI)-derived neuroimaging measures in individuals with T2D; in prior studies T2D affection status has been associated with reduced brain volume, reduced white matter fractional anisotropy, and, less consistently, with increased white matter lesion volume [4-6], all indicative of accelerated brain aging in T2D affected individuals.

Along with presence of T2D, glycemic control and diabetes duration may play roles in variability in neuroimaging measures. Poorer glycemic control in individuals with T2D [7,8] and in the general population [9] is associated with poorer performance on cognitive testing measures, although a recent systematic review showed that associations of glycemic control measures, such as higher fasting glucose and hemoglobin $(\mathrm{Hb}) \mathrm{Alc}$, with poorer cognitive function tend to be weak. HbA1c explained less than $10 \%$ of the variation in cognitive function in most T2D affected cohorts [8]. Some analyses have also shown relationships between glycemic control and cross-sectional brain volumes, progression of brain atrophy, and diffusion tensor imaging measures $[10,8,11,12]$, but results are mixed.

The Diabetes Heart Study Mind (DHS Mind) is a single-center, family-based study which assessed cognitive testing and neuroimaging measures in a population enriched for T2D. Here, we examine associations of neuroimaging measures, including gray (GMV) and white matter volume (WMV), white matter lesion volume (WMLV), diffusion imaging measures, and gray matter cerebral blood flow (GMCBF), with T2D status in European American DHS 
Mind participants. We extended these analyses to assess the relationship of fasting plasma glucose (FPG), HbA1c, and diabetes duration with neuroimaging measures in individuals with T2D.

\section{Methods and Materials}

\section{Study Design and Sample}

Participants in the DHS were recruited from outpatient internal medicine and endocrinology clinics and from the community from 1998 through 2005 in western North Carolina. Siblings affected by T2D without advanced renal insufficiency (serum creatinine concentrations $>2.0 \mathrm{mg} / \mathrm{dl}$ ) were recruited, along with additional non-diabetic siblings. Ascertainment and recruitment have been described [13]. T2D was defined as diabetes developing after the age of 35 years treated with changes in diet and exercise and/or oral agents in the absence of initial treatment solely with insulin and without historical evidence of diabetic ketoacidosis. Diabetes diagnosis was confirmed by review of medications and measurement of FPG and $\mathrm{HbA} 1 \mathrm{c}$ at the exam visit.

The DHS Mind study is an ancillary study to the DHS conducted between 2008 and 2013 [14,15], with pilot subjects recruited beginning in 2005. Cognitive testing and neuroimaging were performed to investigate risk factors for cognitive decline in T2D. Examinations included interviews for medical history and health behaviors, anthropometric measures, assessment of blood pressure, and fasting blood draws. Neuroimaging was successfully performed in 479 participants from the original DHS study examined on average $6.70 \pm 1.53$ years (mean \pm standard deviation (SD)) after their first study visit, and in 305 newly recruited participants. Recruitment criteria in new participants were the same as in the original DHS, except that siblings were not recruited (all new participants were required to have a first-degree relative affected by T2D). Neuroimaging data was available in a total of 784 participants from 514 families, including 682 with T2D from 505 families. FPG and HbA1c values in these analyses were from fasting blood draws at the DHS Mind study visit. FPG was quantified enzymatically using hexokinase, with quantification performed by Wake Forest Baptist Medical Center using a Siemens ADVIA 1650 from August 2005 to June 2008 ( $n=36$ ), by Wake Forest Baptist Medical Center using a Beckman DXC800 from June 2008 to April 2009 ( $\mathrm{n}=83$ ), and by LabCorp from April 2009 to April 2013 using a Roche COBAS $8000 \mathrm{c} 701(\mathrm{n}=665)$. HbA1c was measured using high performance liquid chromatography by Wake Forest Baptist Medical Center using a Bio-Rad VARIANT II TURBO from August 2005 to April 2009 ( $\mathrm{n}=119$ ), with HbA1c measured using immunoassay methods by LabCorp using a Roche COBAS Integra 800A from April 2009 to April 2013 ( $\mathrm{n}=665)$. All analyses were completed in CLIA certified laboratories. Diabetes duration was self-reported by participants from time of diabetes diagnosis to the DHS Mind visit. Participants were examined in the General Clinical Research Center of the Wake Forest Baptist Medical Center. All study protocols were approved by the Institutional Review Board at Wake Forest School of Medicine, and all study procedures were completed in accordance with the Declaration of Helsinki. Participants provided written informed consent prior to participation. 


\section{Neuroimaging}

MR image acquisition-MR imaging was performed on two similar 1.5T GE EXCITE HD scanners with twin-speed gradients using a neurovascular head coil (GE Healthcare, Milwaukee, WI) and on a 3T GE Signa EXCITE scanner, with 769 participants for the 1.5T scanners and 15 participants for the 3T scanner. Imaging protocols have been described in detail previously $[16,17]$. Briefly, gray matter volume (GMV), white matter volume (WMV), and intracranial volume (ICV) (GMV + WMV + cerebrospinal fluid volume (CSFV)) were determined from structural T1 images using the VBM8 toolbox (http://dbm.neuro.unijena.de/vbm.html) automated segmentation procedure, which outputs values for native space total GMV, WMV, and CSFV. Diffusion tensor imaging (DTI) scalar metrics, including fractional anisotropy (FA) and mean diffusivity (MD) in the gray and white matter (GMFA, WMFA, GMMD, and WMMD, respectively), were computed using FSL [18] and the Diffusion Tensor Imaging ToolKit (DTI-TK) (http://www.nitrc.org/projects/dtitk). Cerebral blood flow $(\mathrm{CBF})$ perfusion images were generated using a previously described fully automated data processing pipeline [19], allowing derivation of GMCBF. White matter lesion segmentation was performed using the lesion segmentation toolbox (LST) [20] for SPM8 at a threshold (k) of 0.25, which has been previously validated in DHS Mind [21]. The total white matter lesion volume (WMLV) measure used in these analyses was determined by summing the binary lesion maps and multiplying by the voxel volume.

In total, eight neuroimaging measures were analyzed in this study: GMV, WMV, WMLV, GMCBF, GMMD, WMMD, GMFA, and WMFA. All analyses were adjusted for whether a $1.5 \mathrm{~T}$ or $3 \mathrm{~T}$ scanner was used. All analyses of GMV, WMV, and WMLV included ICV as a covariate.

\section{Statistical Analysis}

Continuous variables were transformed as necessary to approximate normality, including analysis of the natural logarithm of (WMLV+1), FPG, and HbA1c. Relationships between T2D status, diabetes duration, FPG, and $\mathrm{HbA} 1 \mathrm{c}$ and neuroimaging measures were examined using marginal models with generalized estimating equations. The models account for familial correlation using a sandwich estimator of the variance under exchangeable correlation. All participants had data on T2D status $(n=784)$. Among those with T2D $(n=$ 682), 643 had data for FPG, 643 had a HbA1c, and 646 had data on diabetes duration. A Bonferroni correction for multiple comparisons was used, with a threshold of $p<0.0063$ for $a=0.05$ adjusting for the eight traits analyzed. Models were adjusted for covariates as indicated, with Model 1 adjusting for age and scanner (1.5T or 3T) only. Model 2 adjusted for age, scanner, sex, history of cardiovascular disease (CVD), smoking (never, past, current), statin use, and educational attainment (less than high school, high school, greater than high school). Model 3 adjusted for all covariates in Model 2 and blood pressure medication use. Intracranial volume was additionally adjusted for in all models for WMLV, GMV, and WMV. Covariates chosen were similar to those in a similar recent analysis by the ACCORD-MIND study to facilitate comparison of results between the studies [11]. All analyses were performed in SAS 9.3 (SAS Institute, Cary, NC). 


\section{Results}

Table 1 summarizes participant demographic and clinical characteristics, in the full sample $(\mathrm{n}=784$; T2D prevalence $87.0 \%)$, and solely in individuals with T2D ( $\mathrm{n}=682)$. Most individuals were overweight or obese with a high prevalence of hypertension and prior CVD events, as expected for a T2D-enriched cohort. For those with T2D, diabetes duration was $15.2 \pm 7.73$ years (mean $\pm \mathrm{SD}$ (standard deviation)), with a FPG of $146.79 \pm 54.23 \mathrm{mg} / \mathrm{dl}$ and a HbA1c of $7.49 \pm 1.44 \% ; 65.3 \%$ of participants used oral diabetes medications and $33.3 \%$ used insulin. We initially analyzed the association of T2D affection status with eight neuroimaging measures in the DHS Mind cohort. Of these, WMV, GMFA, and WMFA were significantly associated with T2D status after accounting for multiple comparisons ( $\mathrm{p}$ $<0.0063$ ) in models adjusted for age, scanner, sex, history of CVD, smoking, statin use, educational attainment, blood pressure medication use, and, where appropriate, intracranial volume (Table 2). There was also a trend for associations between T2D affected status and higher WMLV ( $\mathrm{p}=0.008)$, higher GMMD ( $\mathrm{p}=0.013)$, and WMMD ( $\mathrm{p}=0.031)$ in Model 3. In Model 1, the associations with higher WMLV $\left(\mathrm{p}=5.20 \times 10^{-4}\right)$ and higher GMMD $(\mathrm{p}=0.001)$ did meet statistical significance. Information on covariate association statistics is available in Supplementary Table 1. T2D status was also analyzed excluded imaging data from the 3T MRI scanner, with similar results (Supplementary Table 2).

In contrast, analyses of FPG, HbA1c, and diabetes duration revealed no significant associations with any neuroimaging measure (Table 3). One nominal association (not meeting our multiple comparisons corrected significance threshold) was observed between diabetes duration and higher GMMD ( $\mathrm{p}=0.04)$ in Model 1, adjusted for only age and scanner. This association was attenuated in Model 2 and Model 3. Information on covariate association statistics is available in Supplementary Table 3. Analyses of FPG, HbA1c, and diabetes duration were repeated excluding imaging data from the 3T MRI scanner, with similar results (Supplementary Table 4).

\section{Discussion}

The association of T2D status and measures of glycemic control with MRI-derived neuroimaging traits, including total brain volume, lesion volume, diffusion tensor imaging measures, and cerebral blood flow, were evaluated in the DHS Mind study. Significant associations of T2D affection status with lower white matter volume and gray and white matter diffusion imaging measures were observed, with nominal association with white matter lesion volume also observed. Among T2D affected participants, HbA1c, FPG, and diabetes duration did not associate with neuroimaging measures. Results suggest that factors other than recent glycemic control may drive changes in cerebral structure in T2D affected DHS Mind participants, with incremental differences in measures such as FPG and HbA1c playing a limited role.

Comparing T2D affected individuals with their non-diabetic siblings in DHS Mind, several neuroimaging measures showed associations with presence of T2D suggestive of accelerated brain aging. The association of T2D with brain volume measures, for example the observed association with total white matter volume $\left(\mathrm{p}=2.48 \times 10^{-6}\right.$ in Model 3$)$, is well established. 
In contrast, analyses of the association of T2D with white matter lesion volume have been less consistent [4-6,22,12]. The significant association observed between T2D and WMLV in Model 1 is notable. The observed association was minimally attenuated by adjustment for smoking and use of hypertensive medications. Smoking and hypertension, as well as increasing age, are widely thought to be significant contributors to development of white matter lesions [23,24]. Similar to most previous reports observing differences in white matter lesion volume with T2D status, the effect size observed in DHS Mind was modest, especially given the wide variation in white matter lesion volume in older adults [4]. We would note that the non-diabetic siblings used as a reference population in DHS-Mind are not traditional healthy controls, with high rates of obesity, hypertension, and CVD, which may minimize the effect sizes observed. Nominal (or better) associations were observed for all diffusion tensor imaging measures assessed, supporting prior reports of white matter fractional anisotropy associating with T2D status $[5,4,12]$.

Despite these associations with T2D, we did not observe any relationships with measures of diabetes severity and glycemic control among those affected by T2D. A recent analysis from the Action to Control Cardiovascular Risk in Diabetes- Memory in Diabetes (ACCORD MIND) study in patients with T2D found that increased FPG and longer diabetes duration were nominally associated with decreased GMV, with longer diabetes duration also associated with increased abnormal white matter volume [11]. We did not replicate these modest associations in our analysis of diabetes duration as a continuous variable or our analysis of similar quartiles of diabetes duration to those used in ACCORD MIND (0-4 years, 5-8 years, 9-14 years, or greater than 15 years). This may be due to differences in sample ascertainment for the DHS Mind, for example recruitment of T2D patients with an HbA1c $<7.5 \%$ and longer mean diabetes duration (15.2 years vs 9.9 years) in the DHS participants, with few DHS participants having short diabetes duration (interquartile range 10.4 to 19.3). Neuroimaging protocols also differ somewhat between the two studies. A systematic review of analyses of glycemic control in patients with T2D found the literature to be mixed on associations with FPG, with some studies finding a weak association between FPG and brain volume and white matter hyperintensities. HbA1c levels were consistently not associated with these neuroimaging measures [8]. Our results support this prior literature indicating that glycemic control measures do not play a major role in variation in neuroimaging measures in T2D. We would note that additional adjustment for oral diabetes medication and insulin use did not change the lack of associations with FPG, $\mathrm{HbA1c}$, and diabetes duration; additional adjustment for the differing methods for HbA1c and FPG assessment also did not substantively change the results for these measures.

While not the focus of the current analysis, we also looked at the cross-sectional cognitive testing measures available in the DHS Mind cohort (Supplementary Tables 5 and 6).

Methods were similar to those for neuroimaging variables. Similar to the neuroimaging results, significant associations with T2D affected status were observed for a number of cognitive testing measures, with T2D associated with lower scores on the Digit Symbol Substitution Task, Rey Auditory Verbal Learning Task, and the Modified Mini-Mental State Examination. In individuals with T2D, longer diabetes duration was associated with lower scores on the Digit Symbol Substitution Task, but no associations with fasting glucose or HbA1C were observed. Our results for both the cognitive testing and neuroimaging 
measures support a model where the presence of diabetes is associated with a consistent modest decrement in age-adjusted cognitive function [25]; however, fasting glucose and $\mathrm{HbA1c}$ measures seem to poorly explain variation within the T2D affected population.

We hypothesize that other risk factors relevant to T2D which have previously been linked to changes in MRI-derived neuroimaging measures, such as hypertension [26], atherosclerosis [27,28], and adiposity [29,30], may be contributing more to variation in these neuroimaging measures in the DHS Mind than diabetes duration or glycemic control. In T2D affected subjects from the DHS cohort, pulse pressure is significantly associated (correcting for multiple comparisons) with white and gray matter volume, as well as cerebral blood flow and fractional anisotropy of the gray and white matter (results not shown). BMI is also associated with gray matter cerebral blood flow in the cohort, suggesting both hypertension and adiposity may play roles in variation in neuroimaging in the DHS Mind. In Model 3 assessing associations of neuroimaging measures with T2D status, history of CVD was at least nominally associated $(p<0.05)$ with all neuroimaging measures except gray matter mean diffusivity, indicating CVD is associated with variation in neuroimaging measures even independent of T2D status. Prior work in the DHS Mind cohort has also found that coronary artery calcification, a measure of subclinical CVD which was assessed at a prior visit, is associated with gray matter volume and nominally with white matter lesion volume as well [17]. Coronary artery calcification was also associated with poorer performance on multiple cognitive tests [14]. Atherosclerosis and CVD burden do seem to be important contributors to variation in cognition and neuroimaging measures in the DHS cohort. Further analysis of these measures in T2D affected cohorts, particularly studies with longitudinal follow-up, is warranted.

The current analysis has a number of strengths. While a number of neuroimaging analyses, notably in ACCORD MIND [11], have analyzed measures of glycemic control for associations with volumetric measures, such as total brain volume and white matter lesion volume, fewer studies have analyzed diffusion tensor imaging and cerebral blood flow measures, an important addition here. ACCORD MIND also does not have non-diabetic controls, preventing an analysis of the effect of diabetes affection status in that cohort [11]. Some prior reports that were able to look at diabetes affected status used a more subjective semi-quantitative scale to assess white matter lesions [22], as opposed to the automated quantitative measure of white matter lesion volume [21] used here, which may increase sensitivity of this measure. The DHS Mind is also a community based cohort, as opposed to a cohort of individuals recruited for a complex clinical trial such as ACCORD MIND, where there are many more participation exclusions. Thus DHS Mind may better reflect the impact of diabetes in the community. Limitations of the current analysis include the cross-sectional nature, the relatively small number of non-diabetic individuals $(n=102)$ for comparative analysis with T2D affection status, and use of a single measure of HbA1c and FPG. It remains possible that glycemic control in prior years might have been associated with brain anatomy, with the most recent measurements failing to reflect earlier diabetes control. Longitudinal assessment of glycemic control, as well as analysis of other glycemic control metrics, including results from oral glucose tolerance tests, postprandial glucose, and measures of hypoglycemia, would be valuable in future studies. The current study also did not include any functional MRI measures, which might be affected by glycemic control in 
T2D patients [31]. Adjustment for different 1.5T scanners used also slightly attenuated the associations with T2D status (results not shown).

In summary, T2D status was associated with brain volumes, differences in fractional anisotropy, and white matter lesion burden in DHS Mind study participants. However, incremental differences in $\mathrm{HbA} 1 \mathrm{c}, \mathrm{FPG}$, and diabetes duration were not associated with neuroimaging measures in patients with T2D in the DHS Mind.

\section{Supplementary Material}

Refer to Web version on PubMed Central for supplementary material.

\section{Acknowledgments}

The authors thank the other investigators, the staff, and the Diabetes Heart Study participants for their valuable contributions.

\section{Funding}

This study was supported in part by the National Institutes of Health through R01 HL67348, R01 HL092301, R01 NS058700 (to DWB), R01 NS075107 (to BIF, JAM), F32 DK083214-01 (to CEH), and F31 AG044879 (to LMR).

\section{References}

1. Lu F-P, Lin K-P, Kuo H-K. Diabetes and the risk of multi-system aging phenotypes: a systematic review and meta-analysis. PLoS ONE. 2009; 4(1):e4144.doi: 10.1371/journal.pone.0004144 [PubMed: 19127292]

2. Reijmer Y, van den Berg E, Ruis C, Kappelle L, Biessels G. Cognitive dysfunction in patients with type 2 diabetes. Diabetes/metabolism research and reviews. 2010; 26(7):507-519. DOI: 10.1002/ dmrr.1112 [PubMed: 20799243]

3. Palta P, Schneider AL, Biessels GJ, Touradji P, Hill-Briggs F. Magnitude of cognitive dysfunction in adults with type 2 diabetes: a meta-analysis of six cognitive domains and the most frequently reported neuropsychological tests within domains. J Int Neuropsychol Soc. 2014; 20(3):278-291. DOI: 10.1017/s1355617713001483 [PubMed: 24555960]

4. Biessels GJ, Reijmer YD. Brain Changes Underlying Cognitive Dysfunction in Diabetes: What Can We Learn From MRI? Diabetes. 2014; 63(7):2244-2252. DOI: 10.2337/db14-0348 [PubMed: 24931032]

5. Falvey C, Rosano C, Simonsick E, Harris T, Strotmeyer E, Satterfield S, Yaffe K, Health ABCS. Macro- and microstructural magnetic resonance imaging indices associated with diabetes among community-dwelling older adults. Diabetes care. 2013; 36(3):677-682. DOI: 10.2337/dc12-0814 [PubMed: 23160721]

6. Moran C, Phan T, Chen J, Blizzard L, Beare R, Venn A, Münch G, Wood A, Forbes J, Greenaway T, Pearson S, Srikanth V. Brain Atrophy in Type 2 Diabetes: Regional distribution and influence on cognition. Diabetes care. 2013; 36(12):4036-4042. DOI: 10.2337/dc13-0143 [PubMed: 23939539]

7. Cukierman-Yaffe T, Gerstein HC, Williamson JD, Lazar RM, Lovato L, Miller ME, Coker LH, Murray A, Sullivan MD, Marcovina SM, Launer LJ. Relationship between baseline glycemic control and cognitive function in individuals with type 2 diabetes and other cardiovascular risk factors: the action to control cardiovascular risk in diabetes-memory in diabetes (ACCORD-MIND) trial. Diabetes care. 2009; 32(2):221-226. DOI: 10.2337/dc08-1153 [PubMed: 19171735]

8. Geijselaers SL, Sep SJ, Stehouwer CD, Biessels GJ. Glucose regulation, cognition, and brain MRI in type 2 diabetes: a systematic review. Lancet Diabetes Endocrinol. 2014; doi: 10.1016/ s2213-8587(14)70148-2 
9. Yaffe K, Blackwell T, Whitmer R, Krueger K, Barrett-Connor E. Glycosylated hemoglobin level and development of mild cognitive impairment or dementia in older women. The journal of nutrition, health \& aging. 2006; 10(4):292-295.

10. van Elderen S, de Roos A, de Craen A, Westendorp R, Blauw G, Jukema J, Bollen E, Middelkoop $\mathrm{H}$, van Buchem M, van der Grond J. Progression of brain atrophy and cognitive decline in diabetes mellitus: a 3-year follow-up. Neurology. 2010; 75(11):997-1002. DOI: 10.1212/WNL. 0b013e3181f25f06 [PubMed: 20837967]

11. Bryan RN, Bilello M, Davatzikos C, Lazar RM, Murray A, Horowitz K, Lovato J, Miller ME, Williamson J, Launer LJ. Effect of Diabetes on Brain Structure: The Action to Control Cardiovascular Risk in Diabetes MR Imaging Baseline Data. Radiology. 2014; 272(1):210-216. DOI: 10.1148/radiol.14131494 [PubMed: 24779562]

12. Weinstein G, Maillard P, Himali JJ, Beiser AS, Au R, Wolf PA, Seshadri S, DeCarli C. Glucose indices are associated with cognitive and structural brain measures in young adults. Neurology. 2015; 84(23):2329-2337. DOI: 10.1212/wnl.0000000000001655 [PubMed: 25948725]

13. Bowden D, Cox A, Freedman B, Hugenschimdt C, Wagenknecht L, Herrington D, Agarwal S, Register T, Maldjian J, Ng M, Hsu F-C, Langefeld C, Williamson J, Carr J. Review of the Diabetes Heart Study (DHS) family of studies: a comprehensively examined sample for genetic and epidemiological studies of type 2 diabetes and its complications. The review of diabetic studies : RDS. 2010; 7(3):188-201. DOI: 10.1900/rds.2010.7.188 [PubMed: 21409311]

14. Hugenschmidt C, Hsu F-C, Hayasaka S, Carr J, Freedman B, Nyenhuis D, Williamson J, Bowden D. The influence of subclinical cardiovascular disease and related risk factors on cognition in type 2 diabetes mellitus: The DHS-Mind study. Journal of diabetes and its complications. 2013; 27(5): 422-428. DOI: 10.1016/j.jdiacomp.2013.04.004 [PubMed: 23659774]

15. Cox AJ, Hugenschmidt CE, Raffield LM, Langefeld CD, Freedman BI, Williamson JD, Hsu FC, Bowden DW. Heritability and genetic association analysis of cognition in the Diabetes Heart Study. Neurobiol Aging. 2014; 35(8):1958.e1953-1958.e1912. DOI: 10.1016/j.neurobiolaging. 2014.03.005 [PubMed: 24684796]

16. Raffield LM, Cox AJ, Hugenschmidt CE, Freedman BI, Langefeld CD, Williamson JD, Hsu F, Maldjian JA, Bowden DW. Heritability and genetic association analysis of neuroimaging measures in the Diabetes Heart Study. Neurobiol Aging. 2015; 36(3):1602.e1607-1615. DOI: 10.1016/ j.neurobiolaging.2014.11.008 [PubMed: 25523635]

17. Hsu FC, Raffield LM, Hugenschmidt CE, Cox A, Xu J, Carr JJ, Freedman BI, Maldjian JA, Williamson JD, Bowden DW. Relationships between Cognitive Performance, Neuroimaging and Vascular Disease: The DHS-MIND Study. Neuroepidemiology. 2015; 45(1):1-11. DOI: 10.1159/000435775 [PubMed: 26185004]

18. Jenkinson M, Beckmann C, Behrens T, Woolrich M, Smith S. FSL. NeuroImage. 2012; 62(2):782790. DOI: 10.1016/j.neuroimage.2011.09.015 [PubMed: 21979382]

19. Maldjian JA, Laurienti PJ, Burdette JH, Kraft RA. Clinical implementation of spin-tag perfusion magnetic resonance imaging. J Comput Assist Tomogr. 2008; 32(3):403-406. DOI: 10.1097/RCT. 0b013e31816b650b [PubMed: 18520545]

20. Schmidt P, Gaser C, Arsic M, Buck D, Forschler A, Berthele A, Hoshi M, Ilg R, Schmid VJ, Zimmer C, Hemmer B, Muhlau M. An automated tool for detection of FLAIR-hyperintense whitematter lesions in Multiple Sclerosis. NeuroImage. 2012; 59(4):3774-3783. DOI: 10.1016/ j.neuroimage.2011.11.032 [PubMed: 22119648]

21. Maldjian J, Whitlow C, Saha B, Kota G, Vandergriff C, Davenport E, Divers J, Freedman B, Bowden D. Automated white matter total lesion volume segmentation in diabetes. AJNR American journal of neuroradiology. 2013; 34(12):2265-2270. DOI: 10.3174/ajnr.A3590 [PubMed: 23868156]

22. van Harten B, de Leeuw F-E, Weinstein H, Scheltens P, Biessels G. Brain imaging in patients with diabetes: a systematic review. Diabetes care. 2006; 29(11):2539-2548. DOI: 10.2337/dc06-1637 [PubMed: 17065699]

23. de Leeuw FE, de Groot JC, Oudkerk M, Witteman JC, Hofman A, van Gijn J, Breteler MM. Hypertension and cerebral white matter lesions in a prospective cohort study. Brain. 2002; 125(Pt 4):765-772. DOI: 10.1093/brain/125.4.765 [PubMed: 11912110] 
24. Longstreth WT Jr, Arnold AM, Beauchamp NJ Jr, Manolio TA, Lefkowitz D, Jungreis C, Hirsch $\mathrm{CH}$, O'Leary DH, Furberg CD. Incidence, manifestations, and predictors of worsening white matter on serial cranial magnetic resonance imaging in the elderly: the Cardiovascular Health Study. Stroke. 2005; 36(1):56-61. DOI: 10.1161/01.str.0000149625.99732.69 [PubMed: 15569873]

25. Biessels GJ, Strachan MW, Visseren FL, Kappelle LJ, Whitmer RA. Dementia and cognitive decline in type 2 diabetes and prediabetic stages: towards targeted interventions. Lancet Diabetes Endocrinol. 2014; 2(3):246-255. DOI: 10.1016/s2213-8587(13)70088-3 [PubMed: 24622755]

26. Schmidt R, Launer L, Nilsson L-G, Pajak A, Sans S, Berger K, Breteler M, de Ridder M, Dufouil C, Fuhrer R, Giampaoli S, Hofman A. Consortium C. Magnetic resonance imaging of the brain in diabetes: the Cardiovascular Determinants of Dementia (CASCADE) Study. Diabetes. 2004; 53(3):687-692. DOI: 10.2337/diabetes.53.3.687 [PubMed: 14988253]

27. Geerlings M, Appelman A, Vincken K, Algra A, Witkamp T, Mali W, van der Graaf Y, Group SS. Brain volumes and cerebrovascular lesions on MRI in patients with atherosclerotic disease. The SMART-MR study. Atherosclerosis. 2010; 210(1):130-136. DOI: 10.1016/j.atherosclerosis. 2009.10.039 [PubMed: 19945704]

28. Vidal JS, Sigurdsson S, Jonsdottir MK, Eiriksdottir G, Thorgeirsson G, Kjartansson O, Garcia ME, van Buchem MA, Harris TB, Gudnason V, Launer LJ. Coronary artery calcium, brain function and structure: the AGES-Reykjavik Study. Stroke. 2010; 41(5):891-897. DOI: 10.1161/strokeaha. 110.579581 [PubMed: 20360538]

29. Debette S, Beiser A, Hoffmann U, Decarli C, O'Donnell CJ, Massaro JM, Au R, Himali JJ, Wolf PA, Fox CS, Seshadri S. Visceral fat is associated with lower brain volume in healthy middle-aged adults. Ann Neurol. 2010; 68(2):136-144. DOI: 10.1002/ana.22062 [PubMed: 20695006]

30. Verstynen T, Weinstein A, Erickson K, Sheu L, Marsland A, Gianaro P. Competing physiological pathways link individual differences in weight and abdominal adiposity to white matter microstructure. NeuroImage. 2013; 79:129-137. DOI: 10.1016/j.neuroimage.2013.04.075 [PubMed: 23639257]

31. He XS, Wang ZX, Zhu YZ, Wang N, Hu X, Zhang DR, Zhu DF, Zhou JN. Hyperactivation of working memory-related brain circuits in newly diagnosed middle-aged type 2 diabetics. Acta Diabetol. 2015; 52(1):133-142. DOI: 10.1007/s00592-014-0618-7 [PubMed: 24993663] 


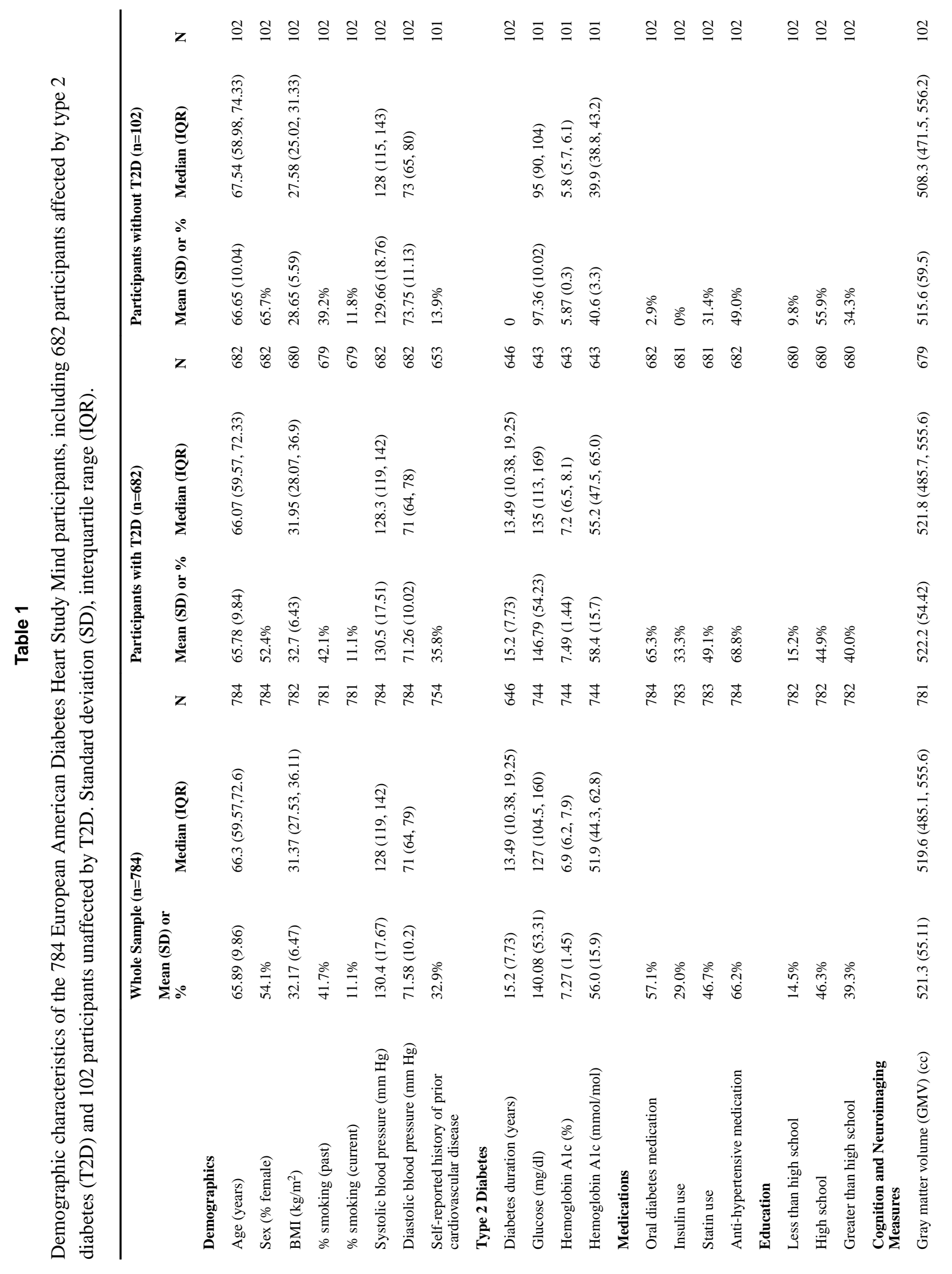




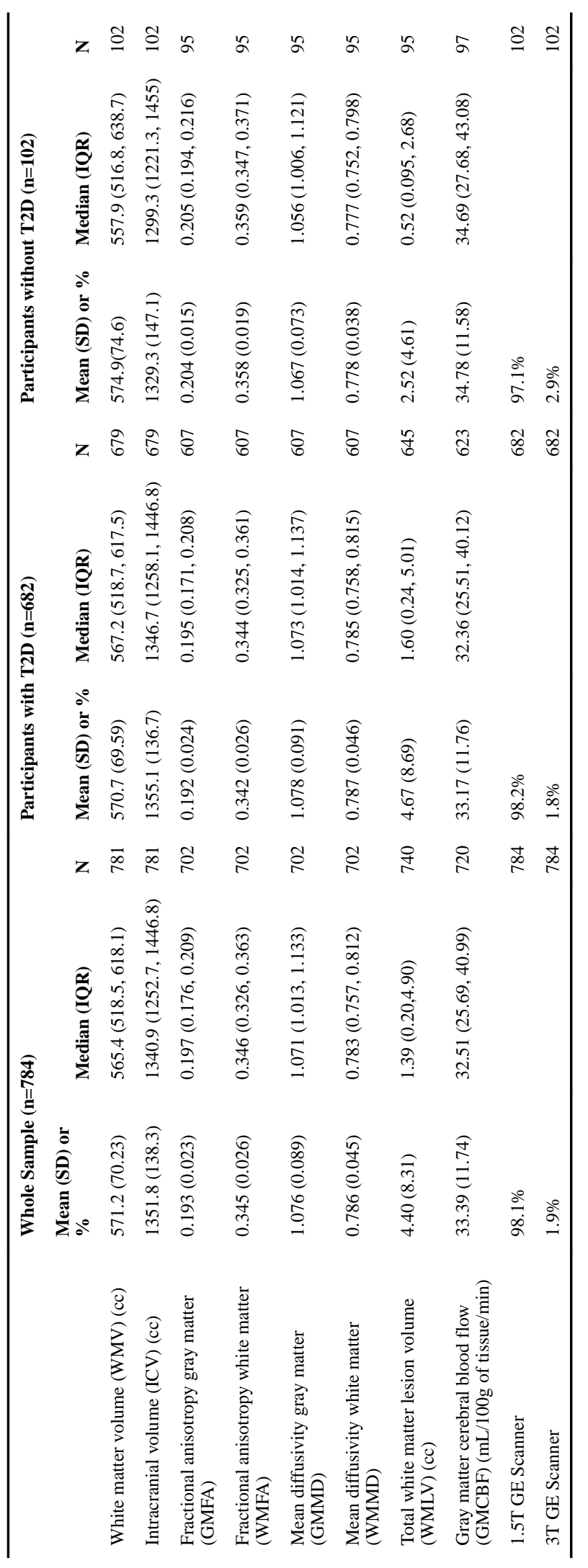


Raffield et al.

Page 13

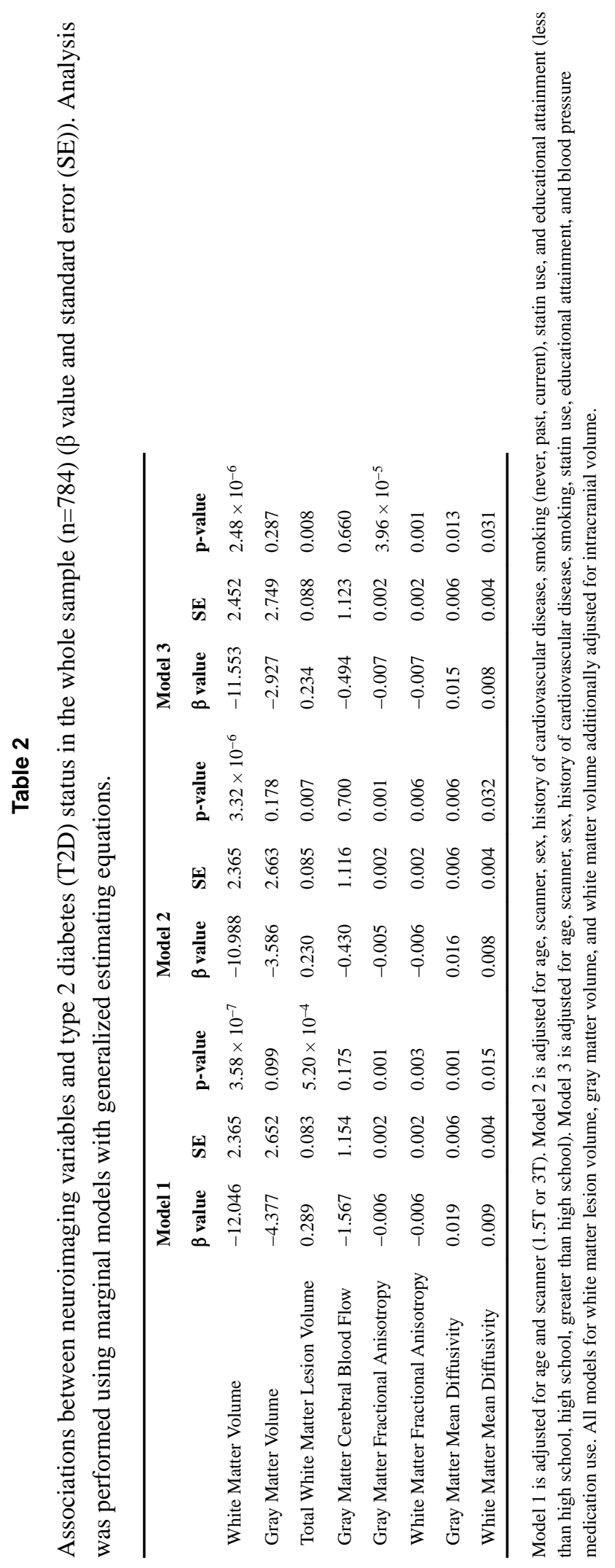


Raffield et al.

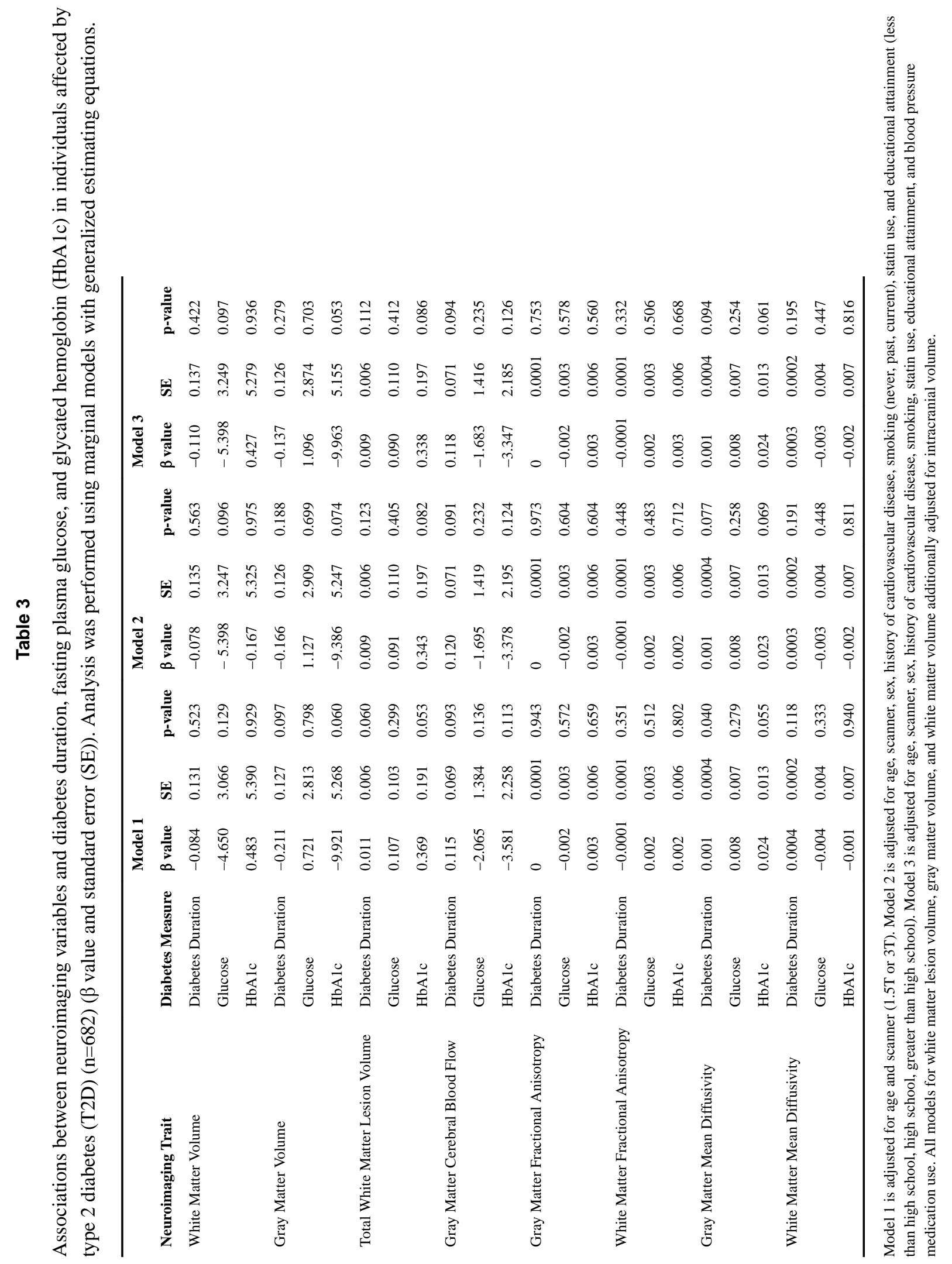

